\title{
Comparação de medidas antropométricas de idosos residentes em área urbana no sul do Brasil, segundo sexo e faixa etária
}

\author{
Anthropometric measures comparison of elderly residents in urban area in southern Brazil by sex \\ and age group
}

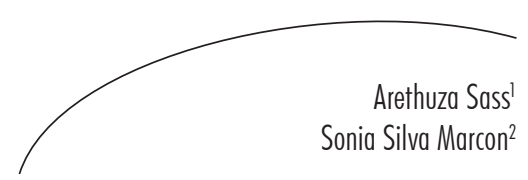

Resumo

Objetivo: Comparar o índice de massa corporal (IMC), dobra cutânea tricipital (DCT), perímetro do braço (PB), circunferência muscular do braço (CMB) e área muscular do braço em idosos (AMB), segundo sexo e faixa etária. Métodos: Estudo transversal realizado com idosos residentes na área urbana de Sarandi-PR, selecionados a partir dos setores censitários e domicílios. Os dados foram coletados no período de outubro de 2010 a março de 2011, com verificação de peso, estatura, PB e DCT e após, calculados IMC, CMB e AMB. Os valores obtidos apresentam-se sob a forma de média, desviopadrão e percentil, com comparação entre os sexos e as faixas etárias. Na análise foram utilizados os testes Qui-quadrado, t Student, ANOVA e Tukey. Diferenças entre os valores médios foram consideradas significativas quando $p<0,05$. Resultados: Os valores médios do IMC (28,80 x 27,26), DCT $(20,93 \times 12,52)$ e PB $(31,92 \times 30,90)$ foram significativamente maiores entre as mulheres, enquanto os homens apresentaram valores médios de CMB (26,97 x 25,35) significativamente maiores. A média da AMB também foi maior entre os homens $(48,97$ x 45,81), mas não de forma significativa. Os valores médios de todas as variáveis diminuíram com o avançar da idade nos dois sexos, com exceção da DCT nos homens. Observou-se, ainda, que a proporção de idosos com obesidade foi significativamente maior entre as mulheres (35,9\% x 24,2\%). Conclusão: Mulheres apresentaram valores médios maiores para a maioria das variáveis antropométricas analisadas. A diminuição nos valores dessas variáveis com o avançar da idade nos dois sexos, inclusive de forma mais acentuada nas mulheres, constitui indicativo da necessidade de monitoramento nutricional e de implementação de estratégias que evitem perdas patológicas na composição corporal de idosos.

\footnotetext{
Hospital Metropolitano, Setor de Nutrição. Sarandi, PR, Brasil.

2 Universidade Estadual de Maringá, Centro de Ciências da Saúde, Departamento de Enfermagem, Programa de Pós-graduação em Ciências da Saúde. Maringá, PR, Brasil.

Palavras-chave: Idoso. Antropometria. Composição Corporal. Estado Nutricional. Diferença entre os Sexos. 


\section{Abstract}

Objective: To compare body mass index (BMI), triceps skinfold (TSF), arm circumference (PB), arm muscle circumference (MCA) and arm muscle area (AMA) in the elderly, according to sex and age group. Methods: Cross-sectional study of elderly residents in urban Sarandi-PR, selected from the census and household sectors. Data were collected from October 2010 to March 2011, assessing weights, height, PB and TSF and after, calculating BMI, MCA and AMA. The values obtained are presented as mean, standard deviation and percentiles, with comparison between genders and age groups. In the analysis we used the chi-square test, t-student, ANOVA and Tukey. Differences between mean values were considered significant when $p<0.05$. Results: The mean values of BMI (28.80 x 27.26), DCT (20.93 x 12.52) and PB (31.92 x 30.90) were significantly higher among women, while men presented average values of CMB (26.97 x 25.35) significantly higher. The average of AMB was also higher among men (48.97 x 45.81), but not significantly. The mean values of all variables decreased. With advancing age in both sexes, except for the DCT in men, it was also observed that the proportion of elderly people with obesity was significantly higher among women (35.9\% x 24.2\%). Conclusion: Women had values higher average for most of the anthropometric variables. The decrease in the values of these variables with advancing age in both sexes, even more steeply in women, is indicative of the need for nutritional monitoring and implementation of strategies to prevent pathological losses body composition in the elderly.
Key words: Elderly. Body Composition. Anthropometry. Nutritional Status.

\section{INTRODUÇÃO}

O envelhecimento é um processo natural, progressivo, caracterizado por alterações físicas, morfológicas, funcionais, psicológicas e sociais que podem levar alterar o estado nutricional e as medidas antropométricas. Com o envelhecimento, ocorre diminuição da massa magra e modificações no padrão de gordura corporal, com aumento de gordura corporal na região do tronco e diminuição nos membros superiores e inferiores, o que leva a variações em indicadores de gordura e massa muscular., ${ }^{1,2}$

A avaliação do estado nutricional é um dos aspectos importantes na avaliação das condições de saúde e bem-estar de idosos. Ela pode ser realizada a partir da utilização de diferentes indicadores antropométricos, de forma isolada ou associada, com o objetivo de identificar a necessidade de correções e intervenções de saúde.

Os indicadores antropométricos também são frequentemente utilizados em pesquisas epidemiológicas, pois o método utilizado para a obtenção dos mesmos é não invasivo, de baixo custo e fácil aplicação. O indicador antropométrico mais conhecido e utilizado em diferentes faixas etárias é o índice de massa corporal (IMC). Contudo, seu uso isolado em idosos é questionável, em função do decréscimo de estatura, acúmulo de tecido adiposo, redução da massa corporal magra e diminuição da quantidade de água no organismo. ${ }^{3}$

Deste modo, outros indicadores antropométricos também são indicados na avaliação do estado nutricional dos idosos, tais como: circunferências do braço (CB), da panturrilha (CP), da cintura (CC) e do quadril (CQ); relação cintura-quadril (RCQ) e dobra cutânea tricipital (DCT). As medidas da CB e $\mathrm{CP}$ permitem estimar a massa muscular do braço e panturrilha, respectivamente, e constituem indicadores de desnutrição em idosos ${ }^{4}$ à medida que identifica a perda da massa muscular. A CC e a RCQ por sua vez, têm sido utilizadas para diagnosticar obesidade abdominal e, consequentemente, risco para doenças cardiovasculares e distúrbios metabólicos, ${ }^{5}$ 
enquanto a DCT é uma forma indireta de mensuração da gordura corporal. ${ }^{4}$ Contudo, a avaliação do estado nutricional de idosos, por meio da antropometria, ainda carece de padrão de referência específico, sendo necessária cautela na comparação antropométrica entre populações distintas. ${ }^{6}$

No Brasil e nos países em desenvolvimento, por exemplo, ainda há poucos estudos referentes aos dados antropométricos da população idosa. A maioria dos dados disponíveis na literatura científica foi produzida com idosos norte-americanos ${ }^{4-7}$ e europeus. ${ }^{8,9}$ A própria Organização Mundial da Saúde $(\mathrm{OMS})^{10}$ relata a necessidade de informações sobre os valores referentes a populações específicas, como é o caso dos idosos.

A inexistência, no Brasil, de um ponto de corte específico para classificar o estado nutricional dos idosos tem desencadeado o uso de diferentes classificações. ${ }^{11-13}$ Os critérios da OMS ${ }^{11}$ por exemplo, são amplamente utilizados em inquéritos nacionais ${ }^{14-16}$ e em estudos realizados no país, 3,17 apesar de sua classificação não ser exclusiva para idosos, sendo adotado os mesmos pontos de corte para adultos. Também tem sido utilizada a classificação proposta por Lipschitz, ${ }^{12}$ que considera as mudanças corporais do indivíduo idoso, sendo os pontos de corte para o baixo-peso e sobrepeso, $22 \mathrm{~kg} / \mathrm{m}^{2}$ e $27 \mathrm{~kg} / \mathrm{m}^{2}$, respectivamente, e o da Organização Pan-americana da Saúde (OPAS), ${ }^{13}$ cujos pontos de cortes são: baixo-peso $\left(\mathrm{IMC} \leq 23 \mathrm{~kg} / \mathrm{m}^{2}\right)$, peso adequado $(23<\mathrm{IMC}<28$ $\left.\mathrm{kg} / \mathrm{m}^{2}\right)$, excesso de peso $\left(28 \leq \mathrm{IMC}<30 \mathrm{~kg} / \mathrm{m}^{2}\right)$ e obesidade (IMC $\geq 30 \mathrm{~kg} / \mathrm{m}^{2}$ ).

Ao optar por utilizar determinado parâmetro, o pesquisador ou profissional precisa estar ciente das diferenças que poderá encontrar e de suas implicações. Os pontos de corte propostos pela $\mathrm{OPAS}^{13}$ e Lipschitz, ${ }^{12}$ por exemplo, são mais sensíveis na identificação do baixo-peso (IMC $\leq 23 \mathrm{~kg} / \mathrm{m}^{2}$ e $<22 \mathrm{~kg} / \mathrm{m}^{2}$, respectivamente) do que os propostos pela OMS (IMC $<18,5$ $\mathrm{kg} / \mathrm{m}^{2} \mathrm{~s}$ ), que, por sua vez, é mais sensível na identificação do excesso de peso, já que considera eutróficas pessoas com IMC até 24,9 $\mathrm{kg} / \mathrm{m}^{2}$, enquanto pelos critérios da $\operatorname{OPAS}^{13}$ e Lipschitz, ${ }^{12}$ IMC até $28 \mathrm{~kg} / \mathrm{m}^{2}$ ou até $27 \mathrm{~kg} / \mathrm{m}^{2}$, respectivamente, ainda caracterizam uma pessoa com peso adequado.

Diante da necessidade de padrões de referência específicos para a população idosa, já que padrões internacionais e referência de indivíduos adultos não são apropriados para serem utilizados em idosos, dada às diferenças corporais entre as populações, o objetivo deste estudo foi comparar o índice de massa corporal (IMC), dobra cutânea triciptal (DCT), perímetro do braço (PB), circunferência muscular do braço (CMB) e área muscular do braço (AMB) em idosos, segundo sexo e faixa etária.

\section{METODOLOGIA}

Estudo de delineamento transversal, de base populacional, realizado com idosos residentes na área urbana de Sarandi-PR, Brasil. Este município tem um índice de desenvolvimento humano (IDH) de 0,768, está localizado na Região Noroeste do Paraná e em $2010^{18}$ tinha uma população de 82.847 habitantes, dos quais 99,15\% residiam na área urbana e 9,51\% (7.884) encontravam-se na faixa etária acima de 60 anos. $^{18}$

Para o cálculo do tamanho da amostra de idosos a serem incluídos no estudo, foram considerados o número (6.527) de idosos residentes na área urbana, ${ }^{19}$ erro de estimativa de $5 \%$, nível de confiança de $95 \%$ e prevalência de $50 \%$ para obter maior variabilidade do evento estudado, acrescido de $20 \%$, para possíveis perdas e/ou recusas, resultado em uma amostra de 436 idosos, porém sete indivíduos foram excluídos porque não foi possível verificar alguma medida antropométrica, de modo que 429 idosos foram efetivamente inseridos no estudo.

A seleção dos idosos em estudo foi realizada a partir dos setores censitários urbanos considerados unidade amostral primária e os domicílios como unidade amostral secundária, por meio de amostragem sistemática com probabilidade 
proporcional ao tamanho do setor. Para tanto, os quarteirões numerados de cada setor censitário, o ponto de início do percurso, assim como a esquina inicial de cada setor foram sorteados, caminhando sempre em sentido horário.

Para proporcionar melhor distribuição a cada domicílio selecionado foi saltado três e quando na casa não tinha o idoso, este era procurado no domicílio seguinte. $\mathrm{Na}$ existência de mais um idoso no domić́lio, foi realizado sorteio simples entre eles.

Os dados foram coletados no período de outubro de 2010 a março de 2011, nos domicílios dos idosos. As medidas antropométricas peso, estatura, PB e DCT foram aferidas por uma única pessoa. $\mathrm{O}$ peso $(\mathrm{kg})$ e a estatura $(\mathrm{m})$ foram mensurados de acordo com as técnicas propostas por Gordon et al. ${ }^{20} \mathrm{O}$ PB e DCT foram aferidas de acordo com as técnicas descritas por Callaway et al. ${ }^{5}$ e Harrison et al., ${ }^{21}$ respectivamente.

Para aferir o peso, utilizou-se balança digital portátil da marca Plenna ${ }^{\circledR}$ modelo Sport, com capacidade de $150 \mathrm{~kg}$ e divisão de 100 gramas. A mensuração da estatura foi realizada utilizando estadiômetro portátil marca Sanny ${ }^{\circledR}$, capacidade máxima de 204 centímetros e precisão de $5 \mathrm{~mm}$. O PB foi aferido com fita métrica inelástica com precisão de $1 \mathrm{~mm}$. A DCT foi realizada com auxílio do adipômetro cientifíco da marca Sanny ${ }^{\circledR}$.

A partir das medidas mensuradas, foram calculados o IMC-peso corporal (kg) dividido pela estatura $(\mathrm{m})$ elevada ao quadrado $\left(\mathrm{kg} / \mathrm{m}^{2}\right)$, CMB obtida pela equação de Gurney \& Jelliffe, ${ }^{19}$ e a AMB obtida a partir das equações específicas por sexo propostas por Heymsfield et al. ${ }^{22}$

$\mathrm{O}$ estado nutricional dos idosos foi determinado apenas segundo o IMC e analisado de acordo com pontos de corte recomendados pela Organização Pan-americana da Saúde (OPAS, 2002), ${ }^{13}$ sendo os idosos classificados com baixo peso $\left(\mathrm{IMC} \leq 23 \mathrm{~kg} / \mathrm{m}^{2}\right)$, peso adequado $\left(23<\mathrm{IMC}<28 \mathrm{~kg} / \mathrm{m}^{2}\right)$, excesso de peso $\left(28 \leq \mathrm{IMC}<30 \mathrm{~kg} / \mathrm{m}^{2}\right)$ e obesidade (IMC $\geq 30 \mathrm{~kg} /$ $\mathrm{m}^{2}$ ). A opção por esse critério se deve ao fato de não existir no Brasil um padrão de referência para idosos e porque os padrões propostos pela OMS referem-se a valores de idosos do continente americano e foi utilizado na avaliação dos idosos que participaram do Projeto SABE Saúde, Bem-estar e Envelhecimento no Brasil. ${ }^{6}$

Foi realizada dupla digitação dos dados, com a finalidade de conferir possíveis inconsistências. Para análise estatística, os idosos foram agrupados de acordo com sexo e grupo etário (60-69 anos, 70-79 anos, 80 anos e mais). As variáveis foram apresentadas em média, desviopadrão (dp) e percentil (P5, P10, P25, P50, P75, P90, P95). A diferença entre as médias e o sexo foi determinada pelo teste $t$ Student. Para verificar o efeito do grupo etário nas variáveis antropométricas, foram realizadas a análise de variância (ANOVA) e a comparação de Tukey. A associação entre o estado nutricional e sexo foi verificada por meio do teste Qui-quadrado. O nível de significância foi fixado em $p<0,05$.

O desenvolvimento do estudo ocorreu em conformidade com o preconizado pela Resolução n 196/96 do Conselho Nacional de Saúde. O projeto foi aprovado pelo Comitê Permanente de Ética em Pesquisa com Seres Humanos da Universidade Estadual de Maringá (parecer $n^{\circ}$ 519/2010). Todos os indivíduos que aceitaram participar do estudo assinaram o Termo de Consentimento Livre e Esclarecido.

\section{RESULTADOS}

Dos 429 idosos em estudo, a maioria era do sexo feminino $(64,33 \%)$. A média de idade foi de 70,75 $\pm 8,12$ anos, sem diferença significativa entre os sexos, sendo média de 70,99 $\pm 8,5$ anos

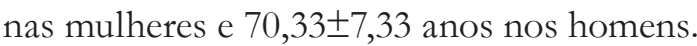

Os valores médios, desvio-padrão, das variáveis antropométricas dos idosos, segundo sexo e grupo etário, estão apresentados na tabela 
1, onde se observa que os valores médios de IMC, CT e PB foram significativamente maiores nas mulheres, enquanto que os da $\mathrm{CMB}$ e da $\mathrm{AMB}$ foram maiores entre os homens, mas apenas na CMB esta diferença foi significativa.
A comparação entre os grupos etários (tabela 1) mostrou tendência de declínio com o avançar da idade nos dois sexos, para todas as variáveis, com exceção da DCT nos homens como para as mulheres.

Tabela 1. Média e desvio-padrão das variáveis antropométricas dos idosos, segundo sexo e grupo etário. Sarandi-PR, 2011.

\begin{tabular}{|c|c|c|c|c|c|c|c|}
\hline \multirow{2}{*}{$\begin{array}{c}\text { Variáveis } \\
\text { antropométricas }\end{array}$} & \multirow[b]{2}{*}{$\mathrm{n}$} & \multicolumn{2}{|c|}{ Homens $(n=153)$} & \multicolumn{3}{|c|}{ Mulheres $(n=276)$} & \multirow[b]{2}{*}{$\mathrm{p}$} \\
\hline & & Média $\pm d p$ & Valor de $p$ & $\mathrm{n}$ & Média $\pm d p$ & Valor de $p$ & \\
\hline $\operatorname{IMC}\left(\mathrm{kg} / \mathrm{m}^{2}\right)$ & & & & & & & $0,002 * *$ \\
\hline $60-69$ & 73 & $28,06 \pm 4,76$ & $0,028(3,653) *$ & 142 & $29,67 \pm 6,38$ & $0,000(7,172)^{*}$ & \\
\hline $70-79$ & 60 & $27,01 \pm 4,20$ & & 83 & $29,02 \pm 5,39$ & & \\
\hline 80 e mais & 20 & $25,13 \pm 3,50$ & & 51 & $26,02 \pm 5,40$ & & \\
\hline Total & 153 & $27,26 \pm 4,47$ & & 276 & $28,80 \pm 6,05$ & & \\
\hline $\mathrm{DCT}(\mathrm{mm})$ & & & & & & & $<0,000^{* *}$ \\
\hline $60-69$ & 73 & $13,23 \pm 6,55$ & $0,172(1,780)$ & 142 & $22,58 \pm 7,97$ & $0,000(12,499)^{*}$ & \\
\hline $70-79$ & 60 & $12,25 \pm 4,36$ & & 83 & $20,70 \pm 6,31$ & & \\
\hline 80 e mais & 20 & $10,73 \pm 3,28$ & & 51 & $16,67 \pm 6,54$ & & \\
\hline Total & 153 & $12,52 \pm 5,45$ & & 276 & $20,93 \pm 7,55$ & & \\
\hline $\mathrm{PB}(\mathrm{cm})$ & & & & & & & $0,033^{* *}$ \\
\hline $60-69$ & 73 & $32,03 \pm 4,47$ & $0,000(10,456)^{*}$ & 142 & $32,99 \pm 5,69$ & $0,000(11,638)^{*}$ & \\
\hline $70-79$ & 60 & $30,68 \pm 3,63$ & & 83 & $32,03 \pm 4,60$ & & \\
\hline 80 e mais & 20 & $27,44 \pm 3,19$ & & 51 & $28,79 \pm 5,43$ & & \\
\hline Total & 153 & $30,90 \pm 4,25$ & & 276 & $31,92 \pm 5,54$ & & \\
\hline $\mathrm{CMB}(\mathrm{cm})$ & & & & & & & $<0,000^{* *}$ \\
\hline $60-69$ & 73 & $27,88 \pm 3,68$ & $0,000(9,749)^{*}$ & 142 & $25,90 \pm 3,81$ & $0,000(7,780)^{*}$ & \\
\hline $70-79$ & 60 & $26,84 \pm 3,30$ & & 83 & $25,52 \pm 3,31$ & & \\
\hline 80 e mais & 20 & $24,06 \pm 2,80$ & & 51 & $23,55 \pm 3,84$ & & \\
\hline Total & 153 & $26,97 \pm 3,62$ & & 276 & $25,35 \pm 3,76$ & & \\
\hline $\operatorname{AMB}\left(\mathrm{cm}^{2}\right)$ & & & & & & & 0,051 \\
\hline $60-69$ & 73 & $52,96 \pm 16,09$ & $0,000(9,474)^{*}$ & 142 & $48,06 \pm 17,19$ & $0,002(6,340)^{*}$ & \\
\hline $70-79$ & 60 & $48,21 \pm 14,49$ & & 83 & $46,24 \pm 13,95$ & & \\
\hline 80 e mais & 20 & $36,71 \pm 10,78$ & & 51 & $38,82 \pm 15,34$ & & \\
\hline Total & 153 & $48,97 \pm 15,69$ & & 276 & $45,81 \pm 16,25$ & & \\
\hline
\end{tabular}

$\mathrm{IMC}=$ índice de massa corporal; $\mathrm{DCT}=$ dobra cutânea tricipital; $\mathrm{PB}=$ perímetro do braço; $\mathrm{CMB}=$ circunferência muscular do braço; $\mathrm{AMB}=$ área muscular do braço; *diferença estatisticamente significativa dos valores médios entre os grupos etários (ANOVA); **diferença estatisticamente significativa dos valores médios entre os sexos ( $t$ Student). 
A análise de comparação múltipla (Test Tukey) demonstrou que houve diferença estatisticamente significativa dos valores médios de IMC, DCT, PB, CMB e AMB, entre os dois grupos de idosos mais jovens (60-69 anos, 70-79 anos) e o grupo etário de 80 anos e mais, com exceção da DCT nos homens.

Na tabela 2, são apresentados os percentis das variáveis antropométricas, os quais mostram, de forma mais detalhada, a diminuição dos valores médios para todas as variáveis em estudo, com o avançar da idade.

A avaliação do estado nutricional dos idosos mostra que uma parcela elevada $(31,7 \%)$ apresentava obesidade e $17,0 \%$ apresentava excesso de peso, com prevalência significativamente maior entre as mulheres (tabela 3).

Tabela 2. Percentis das variáveis antropométricas dos idosos, segundo sexo e grupo etário. Sarandi-PR, 2011.

\begin{tabular}{|c|c|c|c|c|c|c|c|c|}
\hline \multirow{2}{*}{$\begin{array}{l}\text { Variáveis } \\
\text { antropométricas }\end{array}$} & \multirow[b]{2}{*}{$\mathrm{n}$} & \multicolumn{7}{|c|}{ Percentil } \\
\hline & & 5 & 10 & 25 & 50 & 75 & 90 & 95 \\
\hline \multicolumn{9}{|l|}{ IMC $\left(\mathrm{kg} / \mathrm{m}^{2}\right)$} \\
\hline \multicolumn{9}{|l|}{ Mulheres } \\
\hline $60-69$ & 142 & 20,35 & 22,79 & 25,23 & 29,24 & 32,71 & 37,72 & 41,45 \\
\hline 70-79 & 83 & 20,53 & 23,69 & 25,11 & 28,40 & 32,56 & 36,52 & 37,83 \\
\hline 80 e mais & 51 & 17,71 & 18,85 & 22,35 & 25,65 & 29,76 & 33,02 & 35,41 \\
\hline Total & 276 & 19,52 & 21,09 & 24,77 & 28,17 & 32,16 & 36,64 & 40,08 \\
\hline \multicolumn{9}{|l|}{ Homens } \\
\hline $60-69$ & 73 & 20,70 & 23,20 & 25,31 & 27,80 & 30,59 & 34,10 & 35,49 \\
\hline $70-79$ & 60 & 19,95 & 21,47 & 23,76 & 26,77 & 29,78 & 32,92 & 34,45 \\
\hline 80 e mais & 20 & 18,95 & 19,67 & 23,64 & 25,23 & 26,95 & 29,77 & 31,38 \\
\hline Total & 153 & 20,19 & 21,64 & 24,60 & 26,82 & 29,79 & 32,76 & 34,59 \\
\hline \multicolumn{9}{|l|}{$\mathrm{DCT}(\mathrm{mm})$} \\
\hline \multicolumn{9}{|l|}{ Mulheres } \\
\hline $60-69$ & 142 & 9,90 & 12,60 & 17,40 & 22,35 & 27,40 & 31,10 & 35,20 \\
\hline 70-79 & 83 & 10,50 & 11,10 & 16,70 & 20,20 & 25,40 & 29,50 & 31,40 \\
\hline 80 e mais & 51 & 7,50 & 8,80 & 10,70 & 15,90 & 21,20 & 26,00 & 27,10 \\
\hline Total & 276 & 8,80 & 10,50 & 15,90 & 20,50 & 26,05 & 29,60 & 33,00 \\
\hline \multicolumn{9}{|l|}{ Homens } \\
\hline $60-69$ & 73 & 5,10 & 8,00 & 9,90 & 11,50 & 15,20 & 19,50 & 28,80 \\
\hline 70-79 & 60 & 5,95 & 6,70 & 10,00 & 11,80 & 14,45 & 17,25 & 20,20 \\
\hline 80 e mais & 20 & 4,20 & 6,10 & 9,15 & 11,15 & 12,80 & 15,00 & 16,15 \\
\hline Total & 153 & 5,40 & 7,00 & 9,90 & 11,50 & 14,40 & 17,70 & 22,50 \\
\hline
\end{tabular}




\begin{tabular}{|c|c|c|c|c|c|c|c|c|}
\hline \multirow{2}{*}{$\begin{array}{c}\text { Variáveis } \\
\text { antropométricas }\end{array}$} & \multirow[b]{2}{*}{$\mathrm{n}$} & \multicolumn{7}{|c|}{ Percentil } \\
\hline & & 5 & 10 & 25 & 50 & 75 & 90 & 95 \\
\hline \multicolumn{9}{|l|}{$\mathrm{PB}(\mathrm{cm})$} \\
\hline \multicolumn{9}{|l|}{ Mulheres } \\
\hline $60-69$ & 142 & 24,40 & 26,00 & 29,80 & 32,60 & 35,40 & 38,10 & 42,50 \\
\hline 70-79 & 83 & 24,20 & 27,20 & 29,00 & 31,50 & 34,20 & 38,40 & 39,50 \\
\hline 80 e mais & 51 & 20,20 & 22,00 & 24,40 & 28,30 & 32,60 & 35,40 & 39,30 \\
\hline Total & 276 & 23,60 & 24,90 & 28,40 & 31,70 & 34,95 & 38,10 & 41,40 \\
\hline \multicolumn{9}{|l|}{ Homens } \\
\hline $60-69$ & 73 & 23,30 & 27,50 & 29,20 & 32,20 & 34,90 & 38,00 & 39,20 \\
\hline 70-79 & 60 & 24,90 & 26,05 & 28,05 & 30,70 & 33,20 & 35,40 & 38,10 \\
\hline 80 e mais & 20 & 22,20 & 22,65 & 25,05 & 27,70 & 30,05 & 30,75 & 32,20 \\
\hline Total & 153 & 23,30 & 26,40 & 28,20 & 30,60 & 33,30 & 36,10 & 38,40 \\
\hline \multicolumn{9}{|l|}{$\mathrm{CMB}(\mathrm{cm})$} \\
\hline \multicolumn{9}{|l|}{ Mulheres } \\
\hline $60-69$ & 142 & 20,64 & 21,40 & 23,64 & 25,23 & 27,89 & 30,53 & 32,48 \\
\hline 70-79 & 83 & 20,37 & 21,51 & 23,62 & 24,98 & 27,21 & 30,42 & 31,03 \\
\hline 80 e mais & 51 & 18,01 & 19,20 & 20,80 & 23,40 & 25,66 & 27,85 & 31,13 \\
\hline Total & 276 & 20,01 & 20,87 & 22,87 & 24,98 & 27,29 & 30,44 & 32,48 \\
\hline \multicolumn{9}{|l|}{ Homens } \\
\hline $60-69$ & 73 & 20,17 & 23,38 & 25,82 & 28,01 & 30,11 & 31,95 & 34,36 \\
\hline $70-79$ & 60 & 22,18 & 23,15 & 24,43 & 26,40 & 29,12 & 31,18 & 32,88 \\
\hline 80 e mais & 20 & 19,90 & 20,66 & 21,27 & 24,38 & 26,09 & 26,96 & 28,49 \\
\hline Total & 153 & 20,62 & 22,60 & 24,66 & 26,87 & 29,47 & 31,47 & 33,19 \\
\hline \multicolumn{9}{|l|}{$\operatorname{AMB}\left(\mathrm{cm}^{2}\right)$} \\
\hline \multicolumn{9}{|l|}{ Mulheres } \\
\hline $60-69$ & 142 & 27,44 & 29,98 & 38,02 & 45,41 & 55,45 & 67,72 & 77,51 \\
\hline $70-79$ & 83 & 26,56 & 30,36 & 37,93 & 43,21 & 52,48 & 67,19 & 70,68 \\
\hline 80 e mais & 51 & 19,34 & 22,87 & 27,95 & 37,11 & 45,93 & 55,29 & 70,20 \\
\hline Total & 276 & 25,4 & 28,2 & 35,16 & 43,21 & 52,82 & 67,29 & 77,51 \\
\hline \multicolumn{9}{|l|}{ Homens } \\
\hline $60-69$ & 73 & 22,41 & 33,54 & 43,10 & 52,47 & 62,22 & 71,20 & 84,00 \\
\hline $70-79$ & 60 & 29,17 & 32,69 & 37,51 & 45,49 & 57,55 & 67,42 & 76,11 \\
\hline 80 e mais & 20 & 21,59 & 24,00 & 26,04 & 37,34 & 44,22 & 47,88 & 54,82 \\
\hline Total & 153 & 23,86 & 30,68 & 38,41 & 47,51 & 59,16 & 68,88 & 77,72 \\
\hline
\end{tabular}

$\mathrm{IMC}=$ índice de massa corporal; $\mathrm{DCT}=$ dobra cutânea tricipital; $\mathrm{PB}=$ perímetro do braço;

$\mathrm{CMB}=$ circunferência muscular do braço; $\mathrm{AMB}=$ área muscular do braço. 
Tabela 3. Estado nutricional dos idosos, segundo o sexo. Sarandi-PR, 2011.

\begin{tabular}{lccccccc}
\hline \multirow{2}{*}{ Variáveis } & \multicolumn{2}{c}{$\begin{array}{c}\text { Masculino } \\
(\mathrm{n}=153)\end{array}$} & \multicolumn{2}{c}{$\begin{array}{c}\text { Feminino } \\
(\mathrm{n}=276)\end{array}$} & \multicolumn{2}{c}{$\begin{array}{c}\text { Total } \\
(\mathrm{n}=429)\end{array}$} & \multirow{2}{*}{$p^{*}$} \\
\cline { 2 - 6 } & $\mathrm{n}$ & $\%$ & $\mathrm{n}$ & $\%$ & $\mathrm{n}$ & $\%$ & \\
\hline IMC $\left(\mathrm{kg} / \mathrm{m}^{2}\right)$ & 21 & 13,7 & 38 & 13,8 & 58 & 13,8 & \\
Baixo peso & 69 & 45,1 & 92 & 33,3 & 161 & 37,5 & \\
Peso adequado & 26 & 17,0 & 47 & 17,0 & 73 & 17,0 & \\
Excesso de peso & 26 & & & & & & \\
\hline
\end{tabular}

*Diferença estatisticamente significativa (teste Qui-quadrado de Pearson).

\section{DISCUSSÃO}

As alterações físicas (peso e estatura, por exemplo) resultantes do processo de envelhecimento podem influenciar nas dimensões corporais, que quando muito alteradas, interferem na manutenção da saúde, influenciam negativamente na qualidade de vida e na morbimortalidade..$^{23}$ Por essa razão, a avaliação do estado nutricional na população idosa é muito importante. Destaca-se que, nessa população, alterações nutricionais, marcadas pelo baixo-peso, são tão prejudiciais quanto pelo excesso de peso.

Atualmente, o sobrepeso e a obesidade constituem problema de saúde pública e são prevalentes também na população idosa, ${ }^{24} \mathrm{O}$ que tem despertado a atenção dos profissionais de saúde quanto à necessidade de planejamento de estratégias que visem conscientizar a população em geral, quanto aos riscos que essa alteração traz para a saúde em todos os ciclos da vida.

Pesquisas de base populacional, tais como a Pesquisa Nacional de Saúde e Nutrição - PNSN (1989), a Pesquisa sobre Padrões de Vida - PPV (1996/1997) e a Pesquisa de Orçamentos Familiares - POF (2002/2003), há muito tempo vêm demonstrando a inversão nos padrões de distribuição dos problemas nutricionais na população, caracterizando $\mathrm{O}$ processo de transição nutricional, com declínio da prevalência de baixo-peso e aumento da prevalência de sobrepeso e obesidade, inclusive entre os idosos. ${ }^{14-16}$
Pesquisas mais recentes, como a do Projeto SABE e outras realizadas em diferentes regiões do país, também apontam que embora o baixo-peso constitua um problema importante relacionado com o estado nutricional de idosos, a obesidade é muito mais prevalente. ${ }^{3,6}$ No Projeto SABE, a proporção de idosos com peso normal e baixopeso foi maior ( $43 \%$ e $24,1 \%$, respectivamente) do que a encontrada no presente estudo $37,5 \%$ e $13,8 \%$ ), enquanto a prevalência de obesidade e excesso de peso foi menor $(20,8 \%$ e 12,1$),{ }^{6}$ visto ter sido encontrado prevalências de $31,7 \%$ e $17 \%$, respectivamente.

Esses resultados permitem inferir que o estado nutricional dos idosos do presente estudo, marcado pela presença de sobrepeso e obesidade, é pior do que o encontrado no Projeto SABE; 6 contudo, não se pode deixar de considerar que seus dados foram coletados há mais de uma década.

Isto é preocupante, pois além de o excesso de peso constituir um dos fatores de risco mais importante para as doenças não transmissíveis, ${ }^{2}$ com enorme impacto sobre a morbimortalidade, os dados do presente estudo ratificam que a prevalência de excesso de peso em idosos é uma realidade em diferentes contextos e regiões do país. ${ }^{25}$

Destaca-se que os resultados encontrados confirmam a prevalência de obesidade entre idosos do sexo feminino, ${ }^{9}$ uma realidade observada em vários estudos. ${ }^{3,14-16}$ Com relação ao sexo, a PNSN, por exemplo, encontrou prevalência de sobrepeso menor entre os homens $(24,7 \%)$ do 
que entre as mulheres (32\%). Perfil semelhante foi observado na PPV (31,9\% x $32,7 \%$, respectivamente). Já o baixo-peso foi maior no sexo masculino (7,8\% x 6\%), de acordo com a PPV. ${ }^{3,16}$ Nas mulheres, o IMC tende a aumentar até os 75 anos, quando então começa a declinar; e nos homens, o IMC tende a aumentar na meiaidade, estabilizando-se por volta dos 65 anos, declinando a partir dessa idade. ${ }^{11}$

Com relação às comparações múltiplas referentes ao IMC e faixa etária, os resultados do presente estudo corroboram estudo realizado junto a idosos de São Paulo, ${ }^{1}$ em que as diferenças dos valores médios de IMC nos quatro grupos etários mais jovens (60-64, 65-69, 70-74 e 75-79) não foi significativa. Nos homens, os resultados do presente estudo, em que os valores médios de IMC apresentaram diferença estatisticamente significativa entre os grupos etários, corrobora os encontrados nos idosos avaliados no México ${ }^{26}$ e em São Paulo.

O maior acúmulo de gordura nas mulheres, quando comparadas aos homens, é semelhante ao encontrado na literatura., ${ }^{1,2,27}$ Nos idosos residentes no Nordeste, ${ }^{2}$ as mulheres apresentaram valores médios de DCT superiores aos homens correspondendo a 21,3 $\mathrm{mm}$ e $13 \mathrm{~mm}$, respectivamente. Já em São Paulo, ${ }^{6}$ as mulheres apresentaram média de $25,22 \mathrm{~mm}$, e os homens $13,71 \mathrm{~mm}$, ambos valores maiores do que os encontrados no presente estudo.

Os valores de DCT diminuíram com o avançar da idade, o que também é demonstrado em outros estudos. ${ }^{1,2,6}$ Por exemplo, em estudo realizado com idosos residentes em Santiago, Chile, ${ }^{28}$ a diminuição dos valores médios de DCT iniciou-se a partir dos 70 anos. Semelhante ao presente estudo, as idosas do Nordeste $^{2}$ apresentaram diferenças significativas em relação às médias de DCT entre os grupos etários, visto que as mulheres do grupo etário de 60-69 anos apresentaram valores médios $12,2 \%$ maior que das mulheres de 70-79 anos, e 20,9\% maior que das mulheres de 80 anos e mais.

O PB é uma medida que representa o somatório das áreas constituídas pelos tecidos ósseo, muscular, gorduroso e epitelial do braço. Essa medida não é utilizada de forma isolada para verificar a reserva muscular; ${ }^{29}$ entretanto, a diminuição do $\mathrm{PB}$ nos homens está associada à perda de massa muscular, enquanto que, nas mulheres, a perda de tecido adiposo, e ambas, constituem alterações naturais do processo de envelhecimento. ${ }^{4} \mathrm{O} \quad \mathrm{PB}$ apresentou diferença estatisticamente significativa entre os sexos, o que foi observado entre os idosos do Projeto SABE, ${ }^{6}$ mas não entre os idosos do Nordeste ${ }^{2} \mathrm{e}$ nos de Joinvile-SC. ${ }^{30}$

Os valores médios de $\mathrm{PB}$ do presente estudo são inferiores aos encontrados no Nordeste, ${ }^{2}$ onde o valor médio de $\mathrm{PB}$ para homens e mulheres foi o mesmo $(29,3 \mathrm{~cm})$ e superior ao encontrado em São Paulo, ${ }^{6}$ apresentando valores médios de PB de 29,16 cm para homens e 31,02 $\mathrm{cm}$ para mulheres, semelhante aos valores dos idosos em Joinvile-SC. ${ }^{30}$

Em relação aos grupos etários, houve diminuição dos valores médios do PB com o avançar da idade, em ambos os sexos, assim como demonstrado em outros estudos. ${ }^{1,2,7,8,26,27} \mathrm{~A}$ diminuição do PB com o avançar da idade, no estudo com idosos residentes no Nordeste, foi mais pronunciada entre as mulheres. ${ }^{2}$

Com o envelhecimento observa-se, também, declínio na massa corporal magra, ${ }^{1,2}$ sendo CMB e AMB variáveis utilizadas como indicadores de massa muscular. Contudo, o indicador nutricional mais sensível da massa muscular e da reserva protéica, assim como o indicador mais adequado para estimar a subnutrição protéica, é a AMB. ${ }^{11}$ Embora a média com relação a essas variáveis tenha sido maior para os homens, estratégias de manutenção da massa corporal magra necessitam ser desenvolvidas junto às pessoas idosas em geral. Nesses casos, é recomendado o desenvolvimento de programas de treinamento com pesos, adaptados a essa população, promovido e acompanhado por profissionais capacitados. ${ }^{31}$

A variável CMB apresentou diferença estatisticamente significativa em relação ao sexo, com valores médios maiores nos homens. A 
variável AMB também apresentou valores médios superiores nos homens; contudo, não houve diferença significativa em relação ao sexo. Essas variáveis, no estudo com idosos no Nordeste, ${ }^{2}$ apresentaram diferenças estatisticamente significativas, demonstrando maior depósito de massa muscular nos homens.

No presente estudo, homens e mulheres apresentaram diminuição dos valores médios com o avançar da idade das variáveis CMB e AMB, resultado semelhante ao estudo norte-americano, no qual a diminuição com o avanço do grupo etário ocorre para ambos os sexos. ${ }^{7}$ Por outro lado, difere do estudo realizado na Região Nordeste, ${ }^{2}$ em que apenas os homens apresentaram diferença significativa com o avançar da idade.

Os parâmetros antropométricos dos idosos ainda não são compreendidos totalmente $\mathrm{e}$ apresentam variações significativas em diferentes populações. ${ }^{8-17,27}$ Dessa forma, verifica-se a necessidade de padrões de referência específicos, mas até que ocorra a reformulação e validação das metodologias de avaliação e determinação do perfil antropométrico dos idosos, os valores apresentados podem ser utilizados na avaliação clínica e em estudos epidemiológicos do município pesquisado.

Por fim, destacam-se como limitações do estudo a delimitação metodológica constituída por desenho transversal, o qual não permite o estabelecimento de relações de causa e efeito; e o fato de terem sido verificados apenas alguns parâmetros antropométricos, o que não permite uma avaliação global do estado nutricional dos idosos em estudo.

\section{CONCLUSÃO}

Os resultados mostraram diferenças nos valores médios das variáveis antropométricas entre os sexos e tendência de declínio dos valores médios com o avançar da idade A alta prevalência de obesidade na população em estudo, verificada por meio do índice de massa corporal, indica a necessidade de controle e acompanhamento do peso, assim como o desenvolvimento de ações que visem estimular a adesão de hábitos saudáveis de vida.

Nesse sentido, sugere-se a realização de inquéritos abordando os hábitos de vida, bem como a presença de fatores modificáveis, como subsídio para o planejamento de ações efetivas junto aos idosos.

Ressalta-se que a pessoa idosa constitui grupo vulnerável às alterações do estado nutricional, pois além dos declínios naturais advindos da idade, por questões econômicas e sociais, sofre alterações importantes, termos quanti e qualitativos, no consumo alimentar. Por essa razão, ações de vigilância, com caráter multi e interdisciplinar e com enfoque no estado nutricional, são imprescindíveis para a prevenção de distúrbios nutricionais e das doenças a eles relacionadas.

\section{REFERÊNCIAS}

1. Barbosa AR, Souza JMP, Lebrão ML, Laurenti R, Marucci MF. Anthropometry of elderly residents in the city of São Paulo, Brazil. Cad Saúde Pública 2005;21(6):1929-38.

2. Menezes TN, Marucci MFN. Perfil dos indicadores de gordura e massa muscular corporal dos idosos de Fortaleza, Ceará, Brasil Cad Saúde Pública 2007;23(12):2887-95.
3. Silveira EA, Kac G, Barbosa LS. Prevalência e fatores associados à obesidade em idosos residentes em Pelotas, Rio Grande do Sul, Brasil: classificação da obesidade segundo dois pontos de corte do índice de massa corporal. Cad Saúde Pública 2009;25(7):1569-77.

4. Frisancho AR. New standards of weight and body composition by frame size and height for assessment of nutritional status of adults and the eldery. Am J Clin Nutr 1984;40(4):808-19. 
5. Callaway WC, Chumlea WC, Bouchard C, Himes JH, Lohman TG, Martin AD, et al. Circumferences. In: Lohman TG, Roche AF, Martorell R, editors. Anthropometric Standardization Reference Manual. Champaign: Human Kinetics; 1988. p. 39-54.

6. Lebrão ML, Duarte YAO, organizadoras. SABE Saúde, Bem-estar e Envelhecimento - O Projeto Sabe no município de São Paulo: uma abordagem inicial. Brasília, DF: Organização Pan-Americana da Saúde; 2003.

7. Kuczmarski MF, Kuczmarski RJ, Najjar M. Descriptive anthropometric reference data for older Americans. J Am Diet Assoc 2000;100(1):59-66.

8. Delarue J, Constans T, Malvy D, Pradignac A, Couet C, Lamisse F. Anthropometric values in an elderly French population. Brit J Nutr 1994;71(2):295-302.

9. Perissinotto E, Pisent C, Sergi G, Grigoletto F. Anthropometric measurements in the elderly: age and gender differences. Brit J Nutr 2002;87(2):177-86.

10. World Health Organization. Physical status: the use and interpretation of anthropometry. Geneva: World Health Organization;1995. (Technical Report Series, 854).

11. World Health Organization. Obesity: preventing and managing the global epidemic. Geneva: World Health Organization; 1998. (Technical Report Series, 894).

12. Lipschitz DA. Screening for nutritional status in the elderly. Prim Care 1994; 21(1):55-67.

13. Organización Panamericana de la Salud. División de Promoción y Protección de la Salud (HPP). Encuesta Multicentrica salud beinestar y envejecimiento (SABE) em América Latina el Caribe: Informe Preliminar [Internet]. In: XXXVI Reunión del Comité asesor de investigaciones em Salud; 9-11 jun 2001; Kingston, Jamaica: OPAS, 2002 [acesso em 14 fev 2012]. Disponível em: www.opas.org/program/sabe.htm.

14. Tavares EL, Anjos LA. Perfil antropométrico da população idosa brasileira. Resultados da Pesquisa Nacional sobre Saúde e Nutrição. Cad Saúde Pública 1999;15(4):759-68.

15. Campos MAG, Pedroso ERP, Lamounier JA, Colosimo EA, Abrantes MM. Estado nutricional e fatores associados em idosos. Rev Assoc Med Bras 2006;52(4):214-21.

16. Instituto Brasileiro de Geografia e Estatística. Pesquisa de Orçamentos familiares 2002-2003: análise da disponibilidade domiciliar de alimentos e do estado nutricional no Brasil [Internet]. Rio de Janeiro: IBGE; 2004 [acesso em 14 fev 2012]. Disponível em: http://www.ibge.gov.br/home/estatistica/populacao/ condicaodevida/pof/2002analise/pof2002analise.pdf.
17. Cabrera MAS, Wajngarten M, Gebara OCE, Diament J. Relação do índice de massa corporal, da relação cintura-quadril e da circunferência abdominal com a mortalidade em mulheres idosas: seguimento de 5 anos. Cad Saúde Pública 2005;21(3):767-75.

18. Instituto Paranaense de Desenvolvimento Econômico e Social. Caderno Estatístico Município de Sarandi 2011 [Internet]. Sarandi: IPARDES; 2011 [acesso em 14 mar 2012]. Disponível em: http://www.ipardes.gov.br/cadernos/Montapdf. php? Municipio $=86985 \& b t O k=0 k$.

19. Instituto Brasileiro de Geografia e Estatística [Internet]. Rio de Janeiro: IBGE; [1995- ]. Sinopse do censo demográfico 2010; [acesso em 14 mar 2010]; [aproximadamente 2 telas]. Disponível em: http://www.ibge.gov.br/cidadesat/painel/painel. php?codmun=412625\#].

20. Gordon CC, Chumlea WC, Roche AF. Stature, recumbent length, and weight. In: Lohman TG, Roche AF, Martorell R. Anthropometric standardization reference manual. Champaign, Illinois: Human Kinetics Books; 1988. p. 3-8.

21. Harrison GG, Buskirk RE, Carter JEL, Johnston FE, Lohman TG, Pollock ML, et al. Skinfold thicknesses. In: Lohman TG, Roche AF, Martorell $\mathrm{R}$, editors. Anthropometric Standardization Reference Manual. Champaign: Human Kinetics; 1988. p. $55-70$.

22. Heymsfield SB, McManus C, Smith J, Stevens V, Nixon DW. Anthropometric measurement of muscle mass: revised equations for calculating bone-free arm muscle area. Am J Clin Nutr 1982;36(4):680-90.

23. Chumlea WC, Baumgartner RN. Status of anthropometric and body composition data in elderly subjects. Am J Clin Nutr 1989;50(5):1158-66.

24. Da Silva VS, Souza I, Petroski EL, Silva DAS. Prevalência e fatores associados ao excesso de peso em idosos brasileiros. Rev Bras Ativ Fís Saúde 2011;16(4):289-94.

25. Pina FLC, Nascimento MA, Borges Januário RSB, Gerage AM, Oliveira AR, Cyrino ES. Influência da ordem de exercícios com pesos sobre a composição corporal em homens idosos. Rev Educ Fis/UEM 2013;24(3):443-51.

26. Sánchez-García S, Duque-López MX, JuárezCedillo T, Cortés-Núñez AR, Reyes-Beaman S. Anthropometric measures and nutritional status in a healthy elderly population. BMC Public Health [Internet] 2007 [acesso em14 jan 2010];7:1-9. Disponível em: http://www.biomedcentral.com/ content/pdf/1471-2458-7-2.pdf 
27. Santos JL, Albala C, Lera L, García C, Arroyo

P, Pérez-Bravo F, et al. Anthropometric

Measurements in the elderly population of Santiago, Chile. Nutrition 2004;20(5):452-7.

28. Coqueiro RS, Barbosa AR, Borgatto AF. Anthropometric measurements in the elderly of Havana, Cuba: age and sex differences. Nutrition 2009;25:33-9.

29. Frisancho AR. Triceps skinfold and upper arm muscle size norms for assessment of nutritional status. Am J Clin Nutr 1974;27(10):1052-57.
30. Mastroeni MF, Mastroeni SSBS, Erzinger GS, Marucci MFN. Antropometria de idosos residentes no município de Joinville-SC, Brasil. Rev Bras Geriatr Gerontol 2010;13(1):29-40.

31. Silva VS, Souza I, Petroski EL, Silva DAS.

Prevalência e fatores associados ao excesso de peso em idosos brasileiros. Rev Bras Ativ Fís Saúde 2011;16(4):289-93.

Recebido: 10/4/2013

Reapresentado: 22/1/2015

Revisado: 22/2/2015

Aprovado:25/3/2015 